Valderrama, C. (2008). Movimientos sociales: TIC y prácticas políticas. Revista Nómadas. 28, 94-101.

Valencia, A; Arias, M y Vásquez, R. (2009). Ciudadanía y conciencia medioambiental en España. Opiniones y Actitudes. España: CIS

Zibechi,R.(2007).Los movimientossociales comosujetosdela comunicación.América Latina en Movimiento. Recuperado el 20 agosto 2015. Disponible en: http://alainet.org/ resultadobusq.php?page=recherche $\& \mathrm{cx}=001315171428976469354 \% 3 \mathrm{~A} 07 \mathrm{kj} 9 \mathrm{n}$ rjtbi\&cof=FORID\%3A9\&ie=UTF-8\&q=Los+movimientos+sociales+como+sujetos+de+la+comunicaci\%C3\%B3n\&sa=Buscar\&siteurl=http\%3A\%2F\%2Falainet. org\%2Fpublica\%2Frevista.phtml

\section{Los pasos de la memoria en tiempos de la ciudadanía digital}

\author{
Harvey Murcia ${ }^{1}$ \\ Jenny Juliana Jiménez Rodríguez ${ }^{2}$
}

\section{Resumen}

Este capítulo busca mostrar el modo en el que las mutaciones tecnológicas y el paso hacia lo digital han transformado los modos de producción de memoria y de ejercicio de la ciudadanía. Partiendo de la idea de las transformaciones de la percepción y los modos de leer el mundo, buscamos reflexionar sobre las implicaciones de habitar un mundo en el que las certezas de la modernidad parecen ya no estar presentes y donde la construcción del sujeto pasa por nuevas formas de circulación y apropiación de los signos. No pretendemos aquí dar una respuesta definitiva a los modos de producción de la subjetividad contemporánea sino, más bien, abrir el espacio para hacer preguntas que nos permitan comprender nuestro presente.

Palabras claves: memoria, ciudadanía, digitalización, imagen, medios de comunicación

1. Lingüista, y Magíster en Comunicación de la Pontificia Universidad Javeriana. Actualmente es director de la Escuela de Comunicación, Artes visuales y Digitales de la Facultad de Sociedad, Cultura y Creatividad del Politécnico Grancolombiano. Correo electrónico: hmurciaq@poligran.edu.co.

2. Profesional en Estudios Literarios y Magíster en Comunicación de la Pontificia Universidad Javeriana. Actualmente es docente e investigadora de la Facultad de Sociedad, Cultura y Creatividad del Politécnico Grancolombiano. Correo electrónico: jjrodriguez@poligran.edu.co 


\section{Abstract}

This chapter seeks to show the way in which technological mutations and the move towards digital have transformed the modes of production of memory and exercise of citizenship. Starting from the idea of the transformations of perception and ways of reading the world, we seek to reflect on the implications of inhabiting a world in which the certainties of modernity seem no longer present and where the construction of the subject passes through new forms of circulation and appropriation of signs. We do not intend here to give a definitive answer to the modes of production of contemporary subjectivity but, rather, to open the space to ask questions that allow us to understand our present.

Keywords: memory, citizenship, digitization, image, media

\section{Las mutaciones tecnológicas de la experiencia}

La digitalización ha traído consigo numerosos cambios en las formas mismas de la percepción. Como lo intuyeran Benjamin (Benjamin, 2007, 2010) y McLuhan (2002) con las ideas de sensorium y medio, lo clave en el momento de pensar la evolución de las técnicas tiene poco que ver con los aparatos y mucho más con el modo en el que estos afectan la manera de comprender la realidad (Roncallo-Dow, 2012a). Esto fue lo que los llevó a pensar, en el caso de Benjamin en el cine y la fotografía y, con McLuhan nos adentramos en el modo en el que la electricidad trajo una visión implosiva del espacio y el tiempo. Hoy parecemos estar frente a un cambio que, si bien puede y debe ubicarse dentro del mundo eléctrico de McLuhan, ha transformado la percepción y con ella nuestras formas de memoria. Hablamos de la digitalización que, entonces, no implica simplemente una mutación en los soportes sino principalmente un cambio en la legibilidad de la realidad (Blumenberg, 2003).

Siguiendo esta línea de reflexión, Diogo y Sibilia (2017) sostienen que

[en] un mundo en el cual el cambio es la única constante, usar, disfrutar, probar y después descartar la mayor cantidad de cosas posibles puede ser una prueba de riqueza y sagacidad. No sorprende que esa misma lógica se aplique, inclusive, a las fotografías de la propia vida

En esta afirmación parece estar uno de los grandes principios que rigen las formas del ser, el estar y el actuar en el mundo hoy día, en virtud de la permeabilidad de las tecnologías e Internet. Vivimos, o mejor, transitamos vertiginosamente, entre dimensiones actuales y virtuales en las que proliferan servicios y herramientas digitales que atraviesan todos los ámbitos de la sociedad: el político, el económico, el educativo, el artístico, el del ocio, el de las relaciones personales, etc. (Piscitelli, 2002). La forma como nos desenvolvemos en los diferentes contextos está cada vez más subordinada a esa dinámica de usar disfrutar, probar y descartar, en tanto constituye uno de los nuevos valores en la que Castells (Castells, 2005; Castells \& Gimeno, 2004) llamara Sociedad Red, que marcó un cambio decisivo en la ecología mediática contemporánea (Roncallo-Dow \& Scolari, 2016; Carlos Scolari, 2008, 2012; Carlos Scolari, 2015)

Si bien hoy día una gran cantidad de dinámicas sociales están mediadas por lo tecnológico (¿tal vez la mayoría?), las lógicas del ser, estar y actuar no se dan exclusivamente en un entorno virtual, sino que, de hecho, responden a una simbiosis en la que las experiencias se configuran sin límites claros entre lo público y lo privado, lo físico y lo virtual, tal como lo afirma Sibilia (2005):

Tanto la definición como el uso de los espacios sufren alteraciones en función de ese procesamiento digital, que diluye la clásica oposición entre las esferas pública y privada. 
Las subjetividades y los cuerpos contemporáneos se ven afectados por las tecnologías de la virtualidad, y por los nuevos modos que inauguran de entender y vivenciar los límites espaciotemporales que estas tecnologías inauguran (p. 68).

En este punto es importante recordar la reflexión realizada por Eliseo Verón, quien propone que las prácticas que realiza el hombre no pueden ser y entenderse separadas de la tecnología, puesto que, al ser inherentes a su naturaleza, modelan y desarrollan dinámicas y saberes de acuerdo con el subtexto histórico en el que se encuentran. Así, el concepto de "medios designa los usos de las sucesivas tecnologías de comunicación, tal como estos usos se estabilizaron a lo largo de la historia" (Verón, 1996), estableciendo para el individuo otras relaciones con el tiempo, con el espacio y con él mismo.

Y justamente esa necesidad permanente por ajustarnos y amoldarnos a unas nuevas configuraciones del tiempo y el espacio, se evidencia a través de la renovación constante de los ejercicios de la ciudadanía (Fuchs, 2016), los cuales deben adaptarse social y comunicativamente para hacer frente a los movimientos telúricos, casi continuos e incesantes, en lo político, lo económico (Gómez \& Bohórquez, 2018), lo medioambiental (Pellegrini, Puente, \& Grassau, 2015), lo estético (Roncallo-Dow, 2012b), etc.

Estos tiempos, y hablamos en plural porque la categoría de tiempo parece atomizarse y resulta difícil enmarcarse en una época o en una era cuando el cambio es la única constante, se caracterizan por demandar de los individuos unas altísimas competencias de adaptabilidad en diferentes entornos (Anton, 2016; Logan, 2010; McLuhan, 1967, 1996; McLuhan \& Fiore, 2005), sin embargo, las velocidades propias de la convergencia de medios, contenidos, lenguajes, etc. sobrepasan muchas veces las capacidades corporales, intelectuales y emocionales de los sujetos. De ahí que la experiencia, y la asimilación y recuperación de esta se convierta en un proceso sumamente aglomerado, donde la lógica del usar, disfrutar, probar y descartar en el menor tiempo posible, asimila las prácticas de producción, circulación y consumo (Žižek, 2009). “Más allá de "virtualizar" los cuerpos extendiendo su capacidad de acción por el espacio global, la convergencia digital de todos los datos y tecnologías también amplía al infinito las posibilidades de rastreo y colonización de las pequeñas prácticas cotidianas" (Sibilia, 2005, p. 66). Prácticas que desbordan cada vez más los escenarios de la intimidad y transitan lo público haciendo de estas un asunto de la colectividad.

Precisamente en esta colectividad es que aparece el quehacer ciudadano, la participación democrática y los sentidos que se ciernen sobre ella, los cuales no pueden ser pensados fuera de los márgenes de las tecnologías (Ihde, 1990, 2005; Mitcham, 1989); las posibilidades de tejer y fortalecer la sociedad civil están ligadas a las competencias tecnológicas como facilitadoras de lenguajes que permiten la constitución del sujeto como ser político, tal como lo entendía Hannah Arendt (1997), es decir, en tanto actúa de forma concertada con sus semejantes. Este nuevo ciudadano debería estar en capacidad de "utilizar las tecnologías de la información y la comunicación para cuestiones relacionadas con la defensa de sus deberes y derechos tanto políticos como sociales" (Robles, 2009, p.36). Sin embargo, esta cuestión abre un espectro amplísimo de reflexión con relación al marco ético, estético y cultural dentro del cual se hace uso de las tecnologías en el contexto planteado.

De manera pues, que hoy estamos frente a otras formas culturales de estar en el mundo, las cuales trastocan radicalmente el entramado social con el que se alimentan las subjetividades; entonces, las formas de habitar y los tiempos en los que transcurren las acciones, al estar mediados por las tecnologías, proponen pragmáticas y performatividades que son difíciles de fijar, fugaces; condensando las dinámicas de producción, circulación y consumo que están afectando las ciudadanías, sus prácticas y las narrativizaciones de la vida cotidiana (Lipovestky \& Serroy, 2015; Mason, 2017; Zuboff, 2019).

¿Pero por qué pensar y hablar sobre la narrativización de la vida cotidiana? Precisamente porque habitar esgrime un ejercicio propio de la condición cultural: mediatizar lo vivido, como bien lo expone Maldonado (2007)

"Habitar», dijo Benjamin, "es dejar huellas», y las huellas son el sello de la memoria. Como se ha dicho en más de una ocasión, en el mundo occidental la acumulación de objetos vistos como huellas representa uno de los principales medios de definición del propio espacio y de la propia identidad." (p.174)

Así, se podría explicar cómo el proceso del habitar en el tiempo y dejar huella, como lo propone Benjamin, es "necesariamente mediado en todos sus niveles, desde lo micro hasta lo macro, simplemente porque el sentido solo puede circular materializado" (Verón 2013, p. 45), fijado en virtud de alguna técnica propia del momento histórico, siendo consustancial no sólo a las narrativas sino al habitar en sí. Entonces, se delinea una manera particular del dejar huella, que se articula a las dinámicas de producción, circulación y consumo desde la materialización técnica que determina el habitar y las maneras de hacer de la experiencia un relato (Bhabha, 1990).

Estas prácticas hacia las cuales vamos apuntando, son claramente de orden comunicativo, específicamente las que involucran al ciudadano en su configuración digital, asumiendo un rol y unas responsabilidades frente a ciertos procesos de comunicación que sostienen la memoria y posibilitan actos de recordación de la experiencia mediante la na- 
rración. Afirma Walter Benjamin al referirse a este proceso: "La rememoración complementa la 'vivencia'. En ella se precipita la creciente autoalienación del hombre, que hace inventario de todo su pasado como capital ya sin valor. La reliquia procede del cadáver, rememoración de la experiencia ya difunta que, eufemísticamente, se llama vivencia". (Benjamin, 2008) Si bien las experiencias son una secuencia permanente de aconteceres que van expirando a cada instante y yacen muertas en el pasado, estas adquieren el nivel o la categoría de vivencias con la rememoración a través de la narración.

Podemos afirmar entonces, que las prácticas de memoria cumplen pues una función inherente en la construcción del individuo, en la medida que le permite reconocerse de manera tridimensional, en tanto lo que fue, es y será. De modo pues que a través de las prácticas de memoria no solo se reconoce, sino que se representa -para sí y para los otrosen distintos niveles que oscilan entre lo más íntimo y lo público, atravesando diferentes esferas. Como bien lo señaló Salomé Sola "recordar y olvidar son condiciones de posibilidad de la humanización, de la socialización y de la biografía de cada individuo (Ruiz Callejón, 2001: 236). En este sentido, podríamos afirmar que la memoria es coextensiva a los procesos de individuación y a los de socialización” (Sola, 2013, p. 303).

\section{Huellas, relatos y memorias}

Reconociendo pues la memoria como una práctica a través de la cual la experiencia puede recobrar vida una y otra vez mediante el relato, se sobreentiende que este, el relato, tiene múltiples, casi infinitas, posibilidades de ser, y que es según sus particularidades que los "otros" se pueden situar frente a las maneras en que se entiende y se representa un individuo -o un colectivo-. Al tratarse entonces de un relato que se hace y circula en las redes, se expanden las posibilidades de lectura, tal como lo afirma Maldonado: "en el fondo, lo que sugieren los relatos de este ciberespacio es que podemos convertirnos, de flaneurs del espacio interior en flaneurs de la Red". (2007, p. 178).

A pesar de que la red permite expandir de forma permanente las formas de construcción y circulación de los relatos, dentro de este enorme abanico de posibilidades narrativas cabe diferenciar cuatro grandes categorías relativas a la enunciación en términos de las prácticas de la memoria: el individuo que se cuenta a sí mismo -como si fuera otropara recordar; el individuo que le cuenta a otros quién ha sido, qué ha vivido (cómo se ve a sí mismo, qué lugares ha recorrido, con quiénes se ha cruzado en los caminos, qué ha sentido, etc.); el individuo que se recuerda, o se reconoce, a través de las narraciones de otros; y los individuos que hacen un trabajo significativo de memoria de otros que posiblemente no tuvieron la opción de hacerla para sí mismos ni para otros.
¿Pero qué ocurre con las formas de narrar cuando ese habitar ya no solo se da en espacios finitos, con relación a objetos tangibles?, y, sobre todo, ¿cómo se construye la memoria y la identidad por fuera de ese espacio que ya no es propio ni estrictamente privado?. Recordemos que ya hemos señalado cómo el registro y la fijación de experiencias, dadas las naturalezas de tiempo y espacio principalmente en el entorno digital, se complejizan.

Desde siempre, pues, se le ha reconocido a los espacios de vida y a los objetos cotidianos una función primaria como escenario del recuerdo y de la identidad. Desde el Medioevo, «dichos espacios (particularmente el jardín cerrado, la habitación, el corsello [ruelle en francés; pasillo], el estudio y el oratorio), y dichos objetos (el libro, la flor, el vestido, el anillo, la cinta, el retrato y la carta) son totalmente particulares puesto que pertenecieron a un solo individuo, único en el tiempo y en el espacio. Sin embargo, su significado está codificado y es perfectamente comprensible para los demás»". (Alpers, 1983; S. Schama, 1987. en Maldonado, 2007, p. 144).

¿Qué pasa cuando las huellas, esos sellos de la memoria como los llama Benjamin, ya no se dejan solamente en una habitación, sobre las hojas de una libreta, en un álbum familiar etc.? ¿Qué es lo que cambia cuando los escenarios del recuerdo y la identidad no son solo las casas, las habitaciones, los parques, sino todos aquellos rincones que ofrece la red de redes? ¿Cómo es ese habitar y dejar huella a través de la red, o mejor aún, a través de las distintas redes, cada una con sus posibilidades narrativas tan particulares? Todo lo anterior sabiendo que las redes sociales se constituyen en herramientas y espacios a la vez donde el tiempo va tan de prisa, donde las huellas pueden quedar en una nube indefinidamente o desaparecer en un instante.

Pensar en los procesos de memoria, o en las formas de "hacer" memoria a través de redes sociales, implica comprender las formas de producción del habitar, de la subjetividad, el reconocimiento del otro y, por supuesto, de los relatos con los que relaciona su identidad, puesto que todo desciframiento es "un proceso social, en el que toda decodificación e intento de comprensión está determinado histórica y socialmente en la interacción social" (Gutiérrez, 2009, p. 152).

Vale la pena entonces considerar de qué manera se ven impactadas las formas de "dejar huella" tanto del individuo -en su intimidad y en sus diversos roles sociales- como de la colectividad, con la aparición, o mejor aún, invasión y conquista espaciotemporal, por parte de la virtualidad y sus diferentes espectros. Entendiendo acá virtualidad tal como lo hizo Levy (1999), no en oposición a lo real sino a lo actual, sin perder de vista que "las cosas solo tienen límites claros en lo real" (p. 18). 


\section{A modo de conclusión}

Vemos entonces cómo este sincretismo del ser, estar y actuar en el mundo con los límites de lo real, pero a la vez sin los límites de lo virtual, conlleva unas prácticas de memoria que podríamos llamar mixtas, dentro de las cuales se siguen configurando representaciones testimoniales a través de tangibles: como el souvenir que se carga de significado en tanto evidencia de un viaje, por ejemplo. Como representaciones testimoniales de naturaleza virtual que transitan en lo digital, y que a pesar de que permanezcan "allí" indefinidamente y con la potencia de trascender globalmente, tienden a ser más efímeras.

Un claro ejemplo de este último tipo de representación lo podemos encontrar a través de redes sociales como Instagram, que nos hacen pensar en una suerte de relevo del álbum familiar o del portarretratos. No del todo por supuesto, menos ahora que vivimos una época donde en una suerte de resistencia, las prácticas nostálgicas (Boym, 2001; Rubiano, 2013) han elevado su valor, es así como las cámaras polaroid, los casetes, los vinilos, entre otros, se han incorporado con gran fuerza en el mercado cultural. Y cuando hablamos de relevo no lo hacemos en términos equiparables o equitativos, el álbum tiene un carácter prominente de archivo personal (que incluye al colectivo más cercano y se mantiene en la esfera de lo privado), mientras que las prácticas alrededor y dentro de Instagram, si bien pueden atravesar la función del archivo, no se centran allí, principalmente porque propone varios esquemas temporales para la producción, circulación, consumo-interacción. Las dinámicas de esta red facilitan una convergencia de funciones individuales y colectivas que se desarrollan en el ámbito de lo público.

Tal como ya lo hemos señalado, de la mano de los registros análogos, de los objetos simbólicos, de la foto enmarcada tras un vidrio sobre una repisa, conviven esos otros registros que ya son en sí mismos relatos. En Instagram, por ejemplo, ya está dado todo para que se haga un registro enmarcado en unas alteraciones o posibilidades del relato (filtros, marcos, mosaicos, etiquetas, stories, etc.), a la vez que se plantea un escenario inmediato para la interacción: likes, comentarios, envíos, etc. (Manovich, 2017).

Lo anterior no solo nos da pistas relacionadas con la manera en la que estamos construyendo relatos que nos permiten representarnos, sino que nos pone de frente con la cuestión del carácter que le damos a las experiencias, ya que en últimas esto es lo que constituye el impacto, las mutaciones y movimientos en las prácticas de memoria. Los valores significativos y las construcciones simbólicas que se construyen en ese lapso que entendemos como presente, determinarán aquello que se convierta en el pasado, el lugar que este ocupe (en el futuro) y los lazos (afectivos o discursivos) que se puedan establecer con él.

\section{Referencias}

Anton, C. (2016). On the Roots of Media Ecology: A Micro-History and Philosophical Clarification. Philosophies, 1(2). doi: 10.3390/philosophies1020126

Arendt, H. (1997) ¿Qué es política? Barcelona: Paidós.

Benjamin, W. (2007). Sobre la fotografía. Valencia: Pre-textos.

Benjamin, W. (2010). The Work of Art in the Age of Mechanical Reproduction. New York: Pneguin Books.

Bhabha, H. (1990). DisemiNación. Tiempo, narrativa y los márgenes de la nación moderna In H. Bhabha (Ed.), Nación y narración. Entre la ilusión de una identidad y las diferencias culturales (pp. 385-424). Buenos Aires: Siglo XXI.

Blumenberg, H. (2003). Trabajo sobre el mito. Barcelona: Paidós.

Boym, S. (2001). The Future of Nostalgia. New York: Basic Books.

Castells, M. (2005). La sociedad red. Madrid: Alianza Editorial.

Castells, M., \& Gimeno, C. M. (2004). La era de la información: economía, sociedad y cultura: Siglo Veintiuno.

Fuchs, C. (2016). Critical Theory of Communication: New Readings of Lukács, Adorno, Marcuse, Honneth and Habermas in the Age of the Internet. London: University of Westminster Press.

Gómez, C. T., \& Bohórquez, D. N. (2018). When reason does not explain everything: A transnational look at communicative action by citizens in contexts of armed conflict or violence. Palabra Clave, 21(4), 1107-1135. doi:10.5294/pacla.2018.21.4.7

Gutiérrez, E (2009) Leer digital: la lectura en el entorno de las nuevas tecnologías de la información y la comunicación. Signo y Pensamiento XXVIII (54) p. 144-163

Ihde, D. (1990). Technology and the Lifewolrd. From Garden to Earth. Indianapolis: Indiana University Press.

Ihde, D. (2005). La incorporación de lo material: fenomenología y filosofía de la tecnología. Revista iberoamericana de ciencia tecnología y sociedad, 2(5), 153-176. Retrieved from http://www.scielo.org.ar/scielo.php?pid=S1850-00132005000200010\&script=sci_arttext

Jelin, E. y Kaufman, S (2006) Subjetividad y figuras de la memoria. Argentina, Buenos Aires: Siglo XXI Editores.

Lévy, P. (1999) ¿Qué es lo virtual? Barcelona, España: Paidós.

Lipovestky, G., \& Serroy, M. (2015). La estetización del mundo. Vivir en la época del capitalismo artístico. Barcelona: Anagramas.

Logan, R. (2010). Understanding new media: Extending Marshall McLuhan. New York: Peter Lang.

Maldonado, T (2005) Memoria y conocimiento. Sobre los destinos del saber en la perspectiva digital. Barcelona, España: Gedisa.

Manovich, L. (2017). Instagram and Contemporary Image L. Manovich (Ed.) Retrie- 
ved from http://manovich.net/index.php/projects/instagram-and-contemporary-image.

Mason, P. (2017). PostCapitalism: A Guide to Our Future. New York: Farrar, Straus and Giroux.

McLuhan, M. (1967). The invisible environment: The future of an erosion. Perspecta, 11(1), 163-167.

McLuhan, M. (1996). Playboy interview. A candid conversation with the high priest of popcult and metaphysician of media. In E. McLuhan \& F. Zingrone (Eds.), Essential McLuhan (pp. 233-269). New York: Basic Books.

McLuhan, M. (2002). Understanding Media. The Extensions of Man. Cambridge. London: The MIT Press.

McLuhan, M., \& Fiore, Q. (2005). The Medium is the Massage: An Inventory of Effects. New York Penguin Books.

Mitcham, C. (1989). ¿Qué es la filosofía de la tecnología? Madrid: Anthropos.

Pellegrini, S., Puente, S., \& Grassau, D. (2015). La calidad periodística en caso de desastres naturales: cobertura televisiva de un terremoto en Chile. Estudios sobre el Mensaje Periodístico, 21(0). doi:10.5209/rev_ESMP.2015.v21.50678

Piscitelli, A. (2002). meta-Cultura. El eclipse de los medios en la era de Internet. Buenos Aires: La Crujía.

Ramos, D., López, L., Solano, L., Ramírez, J., Beltrán, H., Díaz, W., Morales, M. (2018) La memoria y su devenir en sus espacios: Evidencias del pasado en algunas experiencias cartográficas. (Pensamiento), (palabra)... y obra, \# 20, P. 38-57.

Robles, J (2009) Ciudadanía digital. Una introducción a un nuevo concepto de ciudadano. Barcelona, España: Editorial UOC.

Roncallo-Dow, S. (2012a). Técnica, tecno-logía: más allá de la sinonimia y la objetualidad. Universitas Philosophica, 29(58), 39-65.

Roncallo-Dow, S. (2012b). YouTube como medio aurático. In J. C. Arias, A. Piedrahita, C. Cogua, \& J. A. López (Eds.), Codificar/Decodificar. Prácticas, espacios y temporalidades del audiovisual en Internet (pp. 197-217). Bogotá: Ed. Pontificia Universidad Javeriana.

Roncallo-Dow, S., \& Scolari, C. A. (2016). Marshall McLuhan: The Possibility of Re-Reading His Notion of Medium. Philosophies, 1(2). doi: 10.3390/philosophies1020141

Rubiano, E. (2013). La experiencia sustituida: hacia la construcción tecnológica de la nostalgia. . Palabra Clave, 16(2), 541-558. doi:10.5294/pacla.2013.16.2.11

Scolari, C. (2008). Hipermediacioes. Elementos para una teoría de la comunicación digital interactiva. Barcelona: Gedisa.

Scolari, C. (2012). Media Ecology: Exploring the Metaphor to Expand the Theory. Communication Theory, 22(2), 204-224.

Scolari, C. (2015). From (new)media to (hyper)mediations. Recovering Jesús Martín-Barbero's mediation theory in the age of digital communication and cultural convergence. Information, Communication \& Society, 18(9), 1092-1107. doi:10.108 0/1369118X.2015.1018299

Sibilia, P (2005) El hombre postorgánico. Subjetividad y tecnologías digitales. Argentina Buenos Aires: Fondo de Cultura Económica.

Sibilia, P. y Diogo Ligio (2017) Vitrinas de la intimidad en Internet: ¿Imágenes para guardar o para mostrar?. En Indji, Guido. Silva, Ana (Ed) Clic! Fotografía y Sociedad. Argentina, Buenos Aires: La Marca Editora.

Sola, S. (2013) Memoria mediática y construcción de identidades. Tabula Rasa. Bogotá Colombia, No.19: 301-314, julio-diciembre

Verón E. (1996). La semiosis social 1. Fragmentos de una teoría de la discursividad. Barcelona, España. Gedisa.

Verón E. (2013). La semiosis social 2. Ideas, momentos, interpretantes. Buenos Aires, Argentina: Paidós Estudios de Comunicación.

Žižek, S. (2009). First as Tragedy, then as Farce. Retrieved from https://www.thersa. org/discover/videos/event-videos/2009/11/first-as-tragedy-then-as-farce/

Zuboff, S. (2019). The Age of Surveillance Capitalism: The Fight for a Human Future at the New Frontier of Power. New York: PublicAffairs. 